\title{
Omega-3 and Omega-6 Fatty Acids Kill Thymocytes and Increase Membrane Fluidity
}

\author{
Aparna Prasad ${ }^{1}$, Mari Åhs ${ }^{\S, 2}$, Alexey Goncharov ${ }^{1}$ and David O. Carpenter ${ }^{*}, 1,2$ \\ ${ }^{I}$ Department of Environmental Health Sciences, School of Public Health, University at Albany, Rensselaer, NY 12144, \\ USA \\ ${ }^{2}$ Institute for Health and the Environment, University at Albany, Rensselaer, NY 12144, USA
}

\begin{abstract}
Background: Omega-3 but not omega-6 fatty acids are thought to promote cardiovascular health by increasing membrane fluidity.

Methods: The actions of acute application of omega-3 [docosahexaenoic acid (DHA, 22:6n-3), eicosapentaenoic (EPA, 20:5n-3) and $\alpha$-linolenic acid (ALA, 18:3n-3)] and omega-6 [docosatetraenoic acid (DTA, 22:4n-6), arachidonic acid (ARA, 20:5n-6) and linoleic acid (LNA, 18:2n-6)] fatty acids on plasma membrane fluidity and cytotoxicity were investigated using mouse thymocytes. Membrane fluidity was assessed by determining fluorescence polarization of 1, 6diphenyl-1, 3, 5-hexatriene (DPH) and cell death was assessed by using propidium iodide (PI).

Results: Membrane fluidity in omega-3 treated cells was significantly increased in the order of DHA $>$ EPA $>$ ALA, but DTA and ARA also increased fluidity and were even more potent. Both omega- 3 and omega- 6 fatty acids were acutely cytotoxic to thymocytes at concentrations that altered membrane fluidity, and omega- 6 fatty acids caused more cell death than omega-3s.

Conclusions: The omega- 6 fatty acids, DTA and ARA, are more potent than long chain omega- 3 fatty acids in causing an increase in membrane fluidity in thymocytes.

General Significance: Our results suggest that the beneficial effects of fish consumption are unlikely to be secondary to a selective action of omega-3 fatty acids on membrane fluidity.
\end{abstract}

Keywords: Docosahexaenoic acid, fluorescence polarization, cell death, propidium iodide, flow cytometry, polyunsaturated fatty acids, stearic acid.

\section{INTRODUCTION}

Long chain polyunsaturated fatty acids (PUFAs) are essential in the human diet as humans lack the synthetic enzymes. There are two classes of PUFAs, omega $-3 \mathrm{~s}$ and omega-6s, based on the location of the first double bond in the third (omega-3) or sixth (omega-6) position from the methyl end of the aliphatic carbon chain [1]. Of the omega-3 fatty acids, $\alpha$-linolenic acid (ALA, 18:3n-3) is found in dark green, leafy vegetables, flaxseed, rape seed and walnuts, and can be desaturated and elongated to first form eicosapentaenoic (EPA, 20:5n-3) and then docosahexaenoic acid (DHA, 22:6n-3). However this process is relatively inefficient in humans [2]. Both EPA and DHA are synthesized from ALA by marine microorganisms and accumulate in sea food and fish, which is the primary human source of EPA and DHA. Although seafood is a good source of protein and is low in saturated fats, most beneficial effects

*Address correspondence to this author at the Institute for Health and the Environment, University at Albany, 5 University Place, A 217, Rensselaer, NY 12144, USA; Tel: 518-525-2660; Fax: 518-525-2665;

E-mail: carpent@uamail.albany.edu

${ }^{\S}$ Current Address: Linköping University School of Medicine, Linköping, Sweden of fish consumption and fish oils are usually attributed to the omega-3 PUFAs [3].

The precursor molecule in the omega- 6 family is linoleic acid (LNA, 18:2n-6) which is present in relatively high levels in corn and safflower oils. LNA can be elongated and desaturated to form arachidonic acid (ARA, 20:4n-6) and docosatetraenoic acid (DTA, 22:4n-6). ARA and DTA are primarily found in poultry and meat [2]. ARA is the precursor for some prostaglandins, thromboxane, leukotrienes and other biologically active substances, and therefore omega- 6 fatty acids are considered to be proinflammatory, whereas the omega-3 fatty acids are considered to be anti-inflammatory [4]. Both omega-3 and 6 fatty acids are found in particularly high concentrations in brain, where DHA comprises about 15\%, ARA about $10 \%$ and DTA about $6 \%$ of total brain lipids [5].

Leaf and Xiao [6] reported that in cardiac myocytes omega-3 fatty acids suppress voltage-activated sodium and calcium channels, and showed that the changes in ionic currents result from an alteration in membrane fluidity, which is increased by low-micromolar concentrations of omega-3 fatty acids. Hashimoto et al. [7] found that DHA and EPA also increase membrane fluidity in endothelial cells cultured from the thoracic aorta. The actions of omega-3 
fatty acids on other cell types have not been well studied, and there has been almost no study of the effects of omega- 6 fatty acids on membrane fluidity in any cell type.

Several reports suggest that omega-3 PUFAs decrease the risk of sudden cardiac death following a heart attack [8-10], at least in high-risk populations. Leaf et al. [11] have suggested that the beneficial actions of fish consumption in reducing death after a heart attack are secondary to the ability of omega-3 fatty acids to increase membrane fluidity which then reduces risk of arrhythmias. Thus the question of whether omega- 6 fatty acids also alter membrane fluidity is important.

Some environmental contaminants, such as orthosubstituted polychlorinated biphenyls (PCBs), cause an increase in membrane fluidity in neurons and thymocytes but also cause a rapid cell death in isolated cell systems [12]. Toxaphene, a chlorinated pesticide, decreases membrane fluidity and also causes acute cell death in thymocytes [13]. These observations suggest that membrane fluidity in neurons and thymocytes is tightly regulated and that any sudden perturbation, whether increase or decrease, can result in acute cell death.

The goal of these experiments was to examine the effects of acute administration of omega- 3 and omega- 6 fatty acids on murine thymocytes. We are using thymocytes as a model system for cellular membranes, not because they are immune system cells. If fatty acids exert their beneficial effects on heart muscle by increasing membrane fluidity, and have similar actions on thymocytes, they may also cause acute cell death in thymocytes.

\section{MATERIALS AND METHODS}

\section{Preparation of Thymocytes}

The thymus gland was surgically removed from 4-6 weeks old ICR mice (Taconic Farms Inc., Germantown, NY). The thymus gland was cut into small pieces and thymocytes were gently dissociated in cold Tyrode's buffer using two frosted glass slides as previously described [14]. The cells were filtered through a $70 \mu \mathrm{m}$ cell strainer to remove debris and washed using Tyrode's buffer. The single cell suspension was adjusted to a concentration of approximately $1 \times 10^{6}$ cells/ml using Tyrode's buffer for experiments.

\section{Chemicals and Reagents}

Propidium iodide (PI) was purchased from Molecular Probes Inc. (Eugene, OR). Sodium phosphate monobasic $\left(\mathrm{NaH}_{2} \mathrm{PO}_{4} \cdot \mathrm{H}_{2} \mathrm{O}\right)$ and sodium phosphate dibasic anhydrous $\left(\mathrm{NaH}_{2} \mathrm{PO}_{4}\right)$ were purchased from Fisher Scientific (Fairlawn, NJ). Other chemicals were obtained from Sigma Chemical Co. (St. Louis, MO). Bi-distilled water was used in all the experiments. Fatty acids were dissolved in ethyl alcohol such that the final alcohol concentration was never greater than $0.2 \%$. There was no effect of $0.2 \%$ ethyl alcohol on any of the parameters measured.

\section{Fluorescence Polarization}

Fluorescence polarization was measured on a Perkin Elmer LS50 fluorescence spectrophotometer using 1, 6diphenyl-1,3,5-hexatriene (DPH), a fluorescence probe, to monitor the dynamic behavior of the thymocyte membrane lipid. DPH attaches to the hydrocarbon chains of membrane lipids [15] and the intensity of the fluorescent signal decreases as the ability to move in the lipid layer increases. Membrane fluidity is inversely related to fluorescence polarization; thus, the lower the polarization, the higher the fluidity. A stock solution of DPH was prepared in tetrahydrofuran at $1 \mathrm{mM}$. Cells were mixed with $4 \mu \mathrm{M}$ of DPH and incubated for 30-45 minutes in the dark at room temperature. We used a long incubation time so as to be sure that DPH had equilibrated with intracellular as well as plasma membranes. To measure steady-state fluorescence polarization $(P)$, DPH loaded cells were then incubated with different concentrations of fatty acids for 30 minutes, 1 or 2 hrs at room temperature. The excitation and emission wavelength was set at 360 and $430 \mathrm{~nm}$, respectively $[15,16]$.

\section{Flow Cytometry}

A BD LSRII Flow Cytometer (BD Biosciences, San Jose, CA) was used to measure cell viability. After mixing cells with fluorescent dyes, cells were excited with an argon laser $(488 \mathrm{~nm})$ and 10,000 cells were analyzed per sample. Depending on forward scatter (cell size) and side scatter (cellular granularity), a relatively homogeneous subpopulation of total cells was gated for analysis. Data were analyzed by using BD FACSDiva Software.

To measure cell viability, a DNA binding dye, propidium iodide (PI), was used. After incubating the cells with fatty acids, $10 \mu \mathrm{M}$ PI was added 3-5 minutes before measurement. In healthy cells PI is excluded by the plasma membrane, but in cells with compromised membrane integrity it enters into the cell and binds to DNA. PI fluorescence of live cells is low but in cells with compromised membrane integrity it increases by about 4 orders of magnitude. Each flow cytometry experiment was repeated at least 5 times on separate days.

\section{Statistics}

All results are expressed as a mean \pm S.E.M. Fluorescence polarization and cell viability data relative to control were analyzed with student's t-test. In order to compare cell death and fluidity measures at different concentrations of omega-3 fatty acids we applied one-way ANOVA. One-way ANOVA was also applied to compare effects of ALA and stearic acid on membrane fluidity. For comparison of cell death and fluidity effects of omega-3 as compared to omega-6 fatty acids we used two-way ANOVA. Values were considered to be significant if $\mathrm{p}<0.05$.

\section{RESULTS}

Effects of Omega-3 and Saturated Fatty Acids on Thymocyte Membrane Fluidity

There was no change in membrane fluidity after 30 minutes of incubation with $20 \mu \mathrm{M}$ DHA, EPA or ALA (Fig. 1). However higher concentrations of EPA and DHA $(30 \mu \mathrm{M})$ caused an increased fluidity. With an increase in incubation time ( 1 and $2 \mathrm{hrs}$.) and concentration an increased fluidity was observed with all three omega-3 fatty acids, with DHA showing a greater increase in membrane fluidity than EPA or ALA (Fig. 1). Using a one-way ANOVA the membrane fluidity at $2 \mathrm{hr}$. was significantly different from 


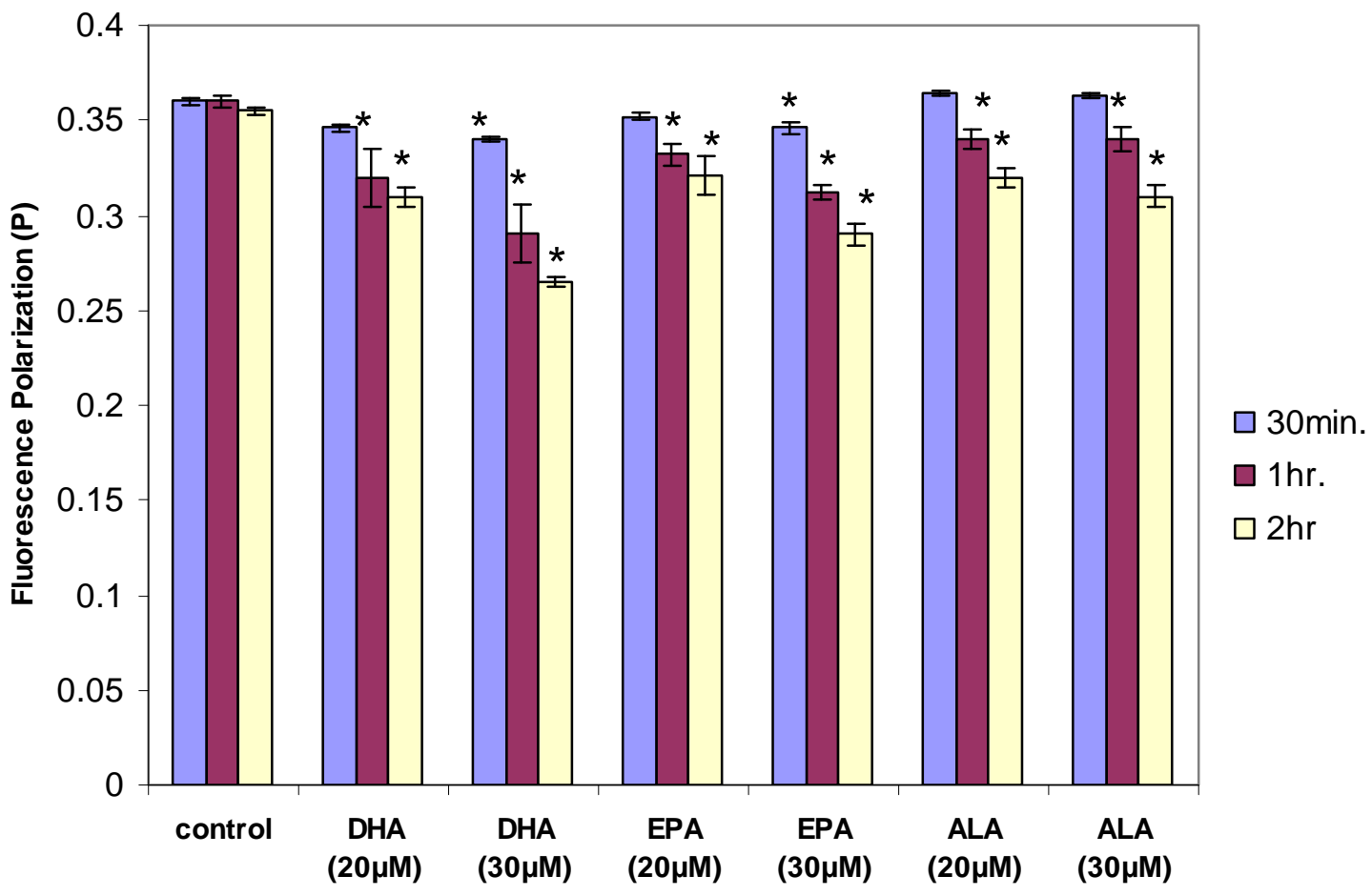

Fig. (1). Effects of omega-3 fatty acids on membrane fluidity as measured by DPH florescence polarization. DPH loaded thymocytes were incubated with DHA, EPA or ALA at 20 and $30 \mu \mathrm{M}$ concentration for 30 minutes, 1 and 2 hrs. DHA (C22) was more effective in increasing membrane fluidity than EPA (C20) or ALA (C18). Lowered florescence reflects an increase in membrane fluidity. * Significantly different from control $(p<0.05)$.

control for all concentrations studied. The membrane fluidity increase due to DHA $(20 \mu \mathrm{M})$ was significantly greater than that due to EPA $(20 \mu \mathrm{M})$, which in turn was significantly greater than that due to ALA $(20 \mu \mathrm{M})$ by one-way ANOVA. In addition DHA $(30 \mu \mathrm{M})$ was significantly different from EPA $(30 \mu \mathrm{M})$ which was significantly different from ALA $(30 \mu \mathrm{M})(\mathrm{p}<0.05)$. Thus in murine thymocytes omega-3 PUFAs increase membrane fluidity in the order of DHA $>$ EPA $>$ ALA.

In contrast stearic acid, a saturated 18-carbon fatty acid, caused a dose-dependent decrease in membrane fluidity after one hour incubation. The magnitude of the decrease in fluidity declined somewhat after 2 hours of incubation, although fluidity was still less than the control. ALA, the 18carbon omega-3 fatty acid, caused an increase in fluidity at both concentrations and time points (Fig. 2). These differences in membrane fluidity change were statistically significant by one-way ANOVA at both concentrations after 2 hours incubation. This result supports the earlier findings that saturated lipids decrease membrane fluidity [17], whereas omega-3 fatty acids cause an increase in fluidity.

\section{Effects of Omega-6 Fatty Acids on Thymocyte Membrane Fluidity}

The long chain omega-6 PUFAs also increased membrane fluidity. By two-way ANOVA the increase in membrane fluidity was significantly greater for DTA and ARA than for DHA and EPA at both concentrations $(\mathrm{p}<0.05)$ The C-22 omega-6 fatty acid (DTA) was more effective in increasing fluidity than the C-20 omega- 6 fatty acid (ARA) (Fig. 3). In contrast to DTA and ARA, LNA decreased membrane fluidity. As with stearic acid, the decrease declined somewhat with longer incubation times.

\section{Effects of Omega-3 and Saturated Fatty Acids on Cell Viability}

Thymocytes were treated with DHA, EPA or ALA at different concentrations and incubation times and PI fluorescence measured in the flow cytometer. For all three fatty acids, concentrations and incubation times that resulted in an increase in membrane fluidity also caused acute cell death of thymocytes. The degree of cell death increased with concentration and incubation time in the order of DHA $>$ EPA $>$ ALA (Fig. 4). By one-way ANOVA DHA at 20 and $30 \mu \mathrm{M}$ was significantly more potent than EPA at the same concentrations, which in turn was significantly more potent that ALA at these concentrations $(p<0.05)$. Stearic acid caused only a very small decrease in thymocyte cell viability (Fig. 5).

\section{Effects of Omega-6 Fatty Acids on Cell Viability}

Similar studies were done to monitor acute cytotoxicity of omega- 6 fatty acids in the concentration ranges that also increase membrane fluidity. Thymocytes were incubated with DTA, ARA or LNA at different concentrations and incubation times. DTA was extremely toxic to thymocytes, killing nearly $100 \%$ of cells at the higher concentrations. DTA was more toxic and caused more cell death than ARA with increasing concentrations and incubation times (Fig. 6) $(\mathrm{p}<0.05$ by two-way ANOVA). Thus in this assay system C22 and C-20 omega- 6 fatty acids are more toxic to cells than omega-3 fatty acids. The C18 omega- 6 fatty acid (LNA), 


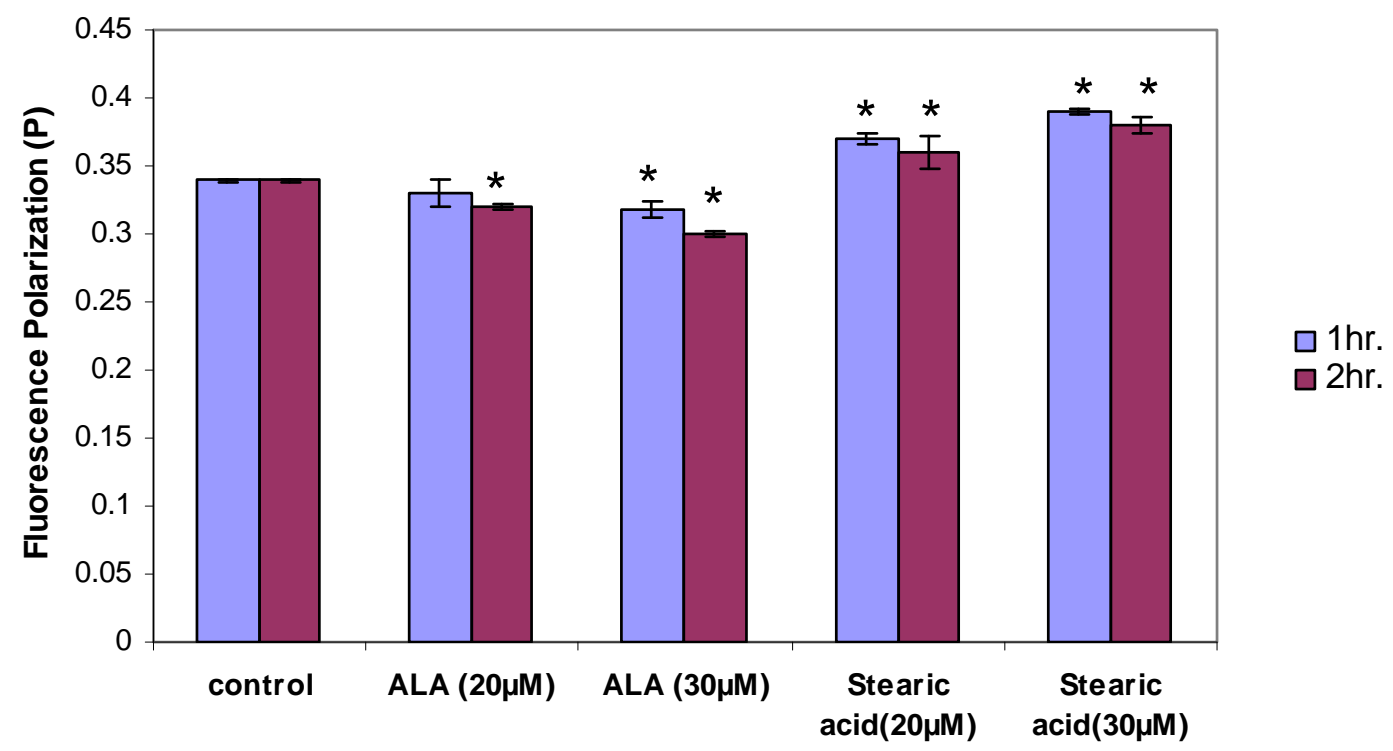

Fig. (2). Effects of ALA and stearic acid on membrane fluidity as measured by DPH florescence polarization. DPH loaded thymocytes were incubated with ALA or stearic acid $(20$ and $30 \mu \mathrm{M})$ for 1 and 2 hrs. While ALA increased membrane fluidity, the saturated stearic acid caused a decrease in membrane fluidity. Increased florescence reflects a decrease in membrane fluidity. ${ }^{*}$ Significantly different from control $(\mathrm{p}<0.05)$.

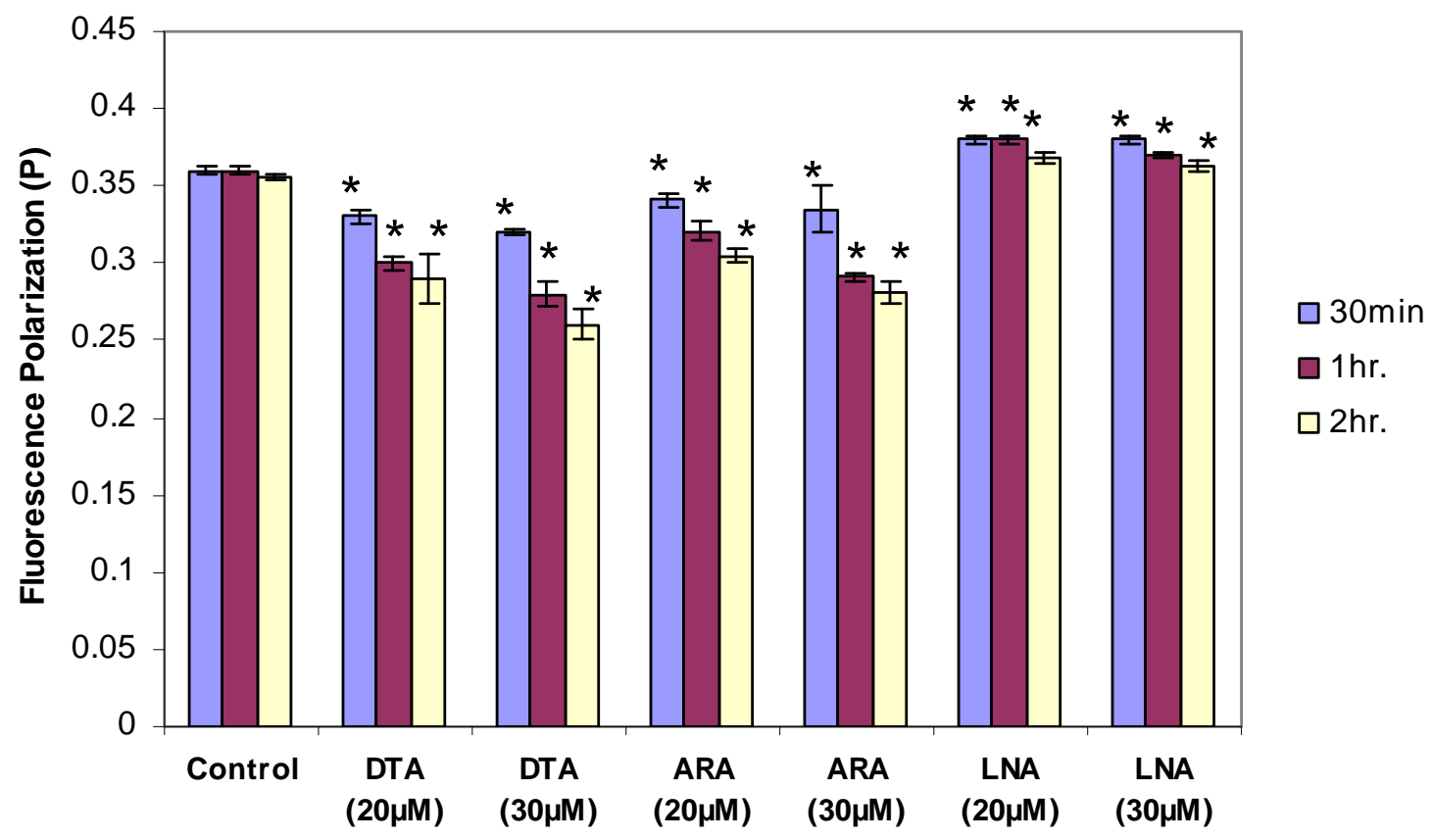

Fig. (3). Effect of omega-6 fatty acids on membrane fluidity as measured by DPH florescence polarization. DPH loaded thymocytes were incubated with DTA, ARA or LNA $(20$ and $30 \mu \mathrm{M})$ for $30 \mathrm{~min}, 1$ and $2 \mathrm{hrs.} \mathrm{DTA} \mathrm{(C22)} \mathrm{and} \mathrm{ARA} \mathrm{(C20)} \mathrm{increased} \mathrm{but} \mathrm{LNA} \mathrm{(C18)} \mathrm{decreased}$ membrane fluidity. * Significantly different from control $(\mathrm{p}<0.05)$.

which decreased rather than increased membrane fluidity, was even more toxic to cells at all concentrations and incubation times than either DTA or ARA. Even at a concentration of $10 \mu \mathrm{M}$ almost all cells were dead within 1.5 $\mathrm{hr}$.

\section{DISCUSSION}

Our studies show a general pattern in which acute application of PUFAs resulted in an increase in membrane fluidity of murine thymocytes. The potency at increasing membrane fluidity was well correlated with the ability to cause acute cell death. The omega-6s were somewhat more potent than the omega-3s, and the longer the PUFA carbon chain the greater the effect. The exception to this pattern was LNA, which surprisingly caused a decrease in fluidity, but also was more potent than any of the other PUFAs tested in causing cytotoxicity. Further study is necessary to 


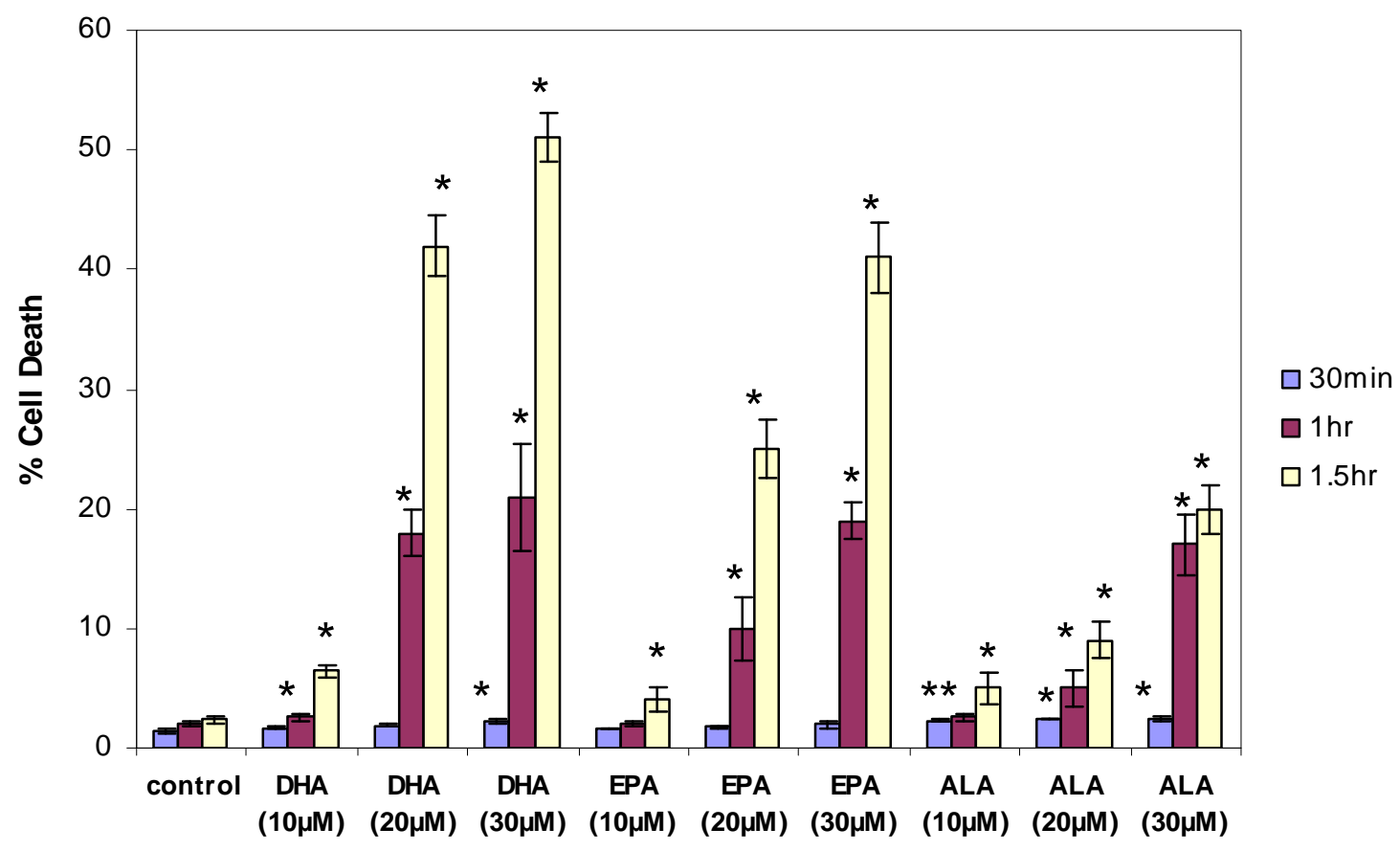

Fig. (4). Acute cell death, measured as PI florescence, induced in thymocytes after exposure to omega-3 fatty acids. Thymocytes were incubated with DHA, EPA or ALA for $30 \mathrm{~min}, 1$ and $1.5 \mathrm{hrs}$ with various concentrations. * Significantly different from control $(\mathrm{p}<0.05)$.

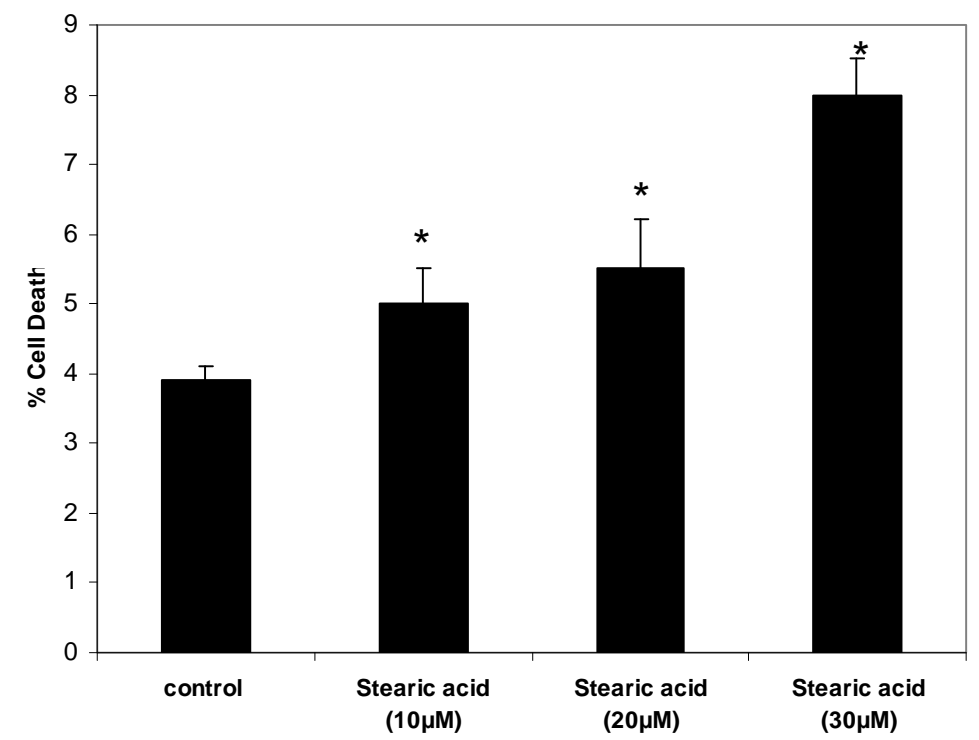

Fig. (5). Cell death resulting from exposure to stearic acid. Thymocytes were incubated with different concentrations of stearic acid for one hour and cell death was measured as PI iodide florescence. Note that the scale for cell death is different from that in Fig. (4). *Significantly different from control $(\mathrm{p}<0.05)$.

understand why LNA acts differently. Saturated fatty acids decreased fluidity, but caused little cell death.

The physiological implications of our observations are not clear, primarily because in these experiments we are acutely altering membrane structure. There is no evidence that dietary ingestion of these lipids results in a sufficient rapid change in concentration so as to alter membrane fluidity and trigger cell death. There are clearly regulatory mechanisms controlling fluidity in different membranes, as reflected by the fact that omega- 3 fatty acids concentrate in neuronal membranes to a much greater degree than in other types of cells. However several other in vitro studies have shown that DHA and related PUFAs induce cell death by apoptosis in a variety of tumor cell lines. Sturlan et al. [18] reported that DHA reduced tumor cell viability at $25 \mu \mathrm{m}$. Siddiqui et al. [19] reported that at $20 \mu \mathrm{m}$ DHA causes a rapid increase in cell death in cancer cells. These cell culture effects are most likely secondary to altered membrane fluidity.

The increase in fluidity of cardiac muscle membranes by omega-3 fatty acids has been proposed to be the basis for a lowered probability that arrhythmias will result following a 


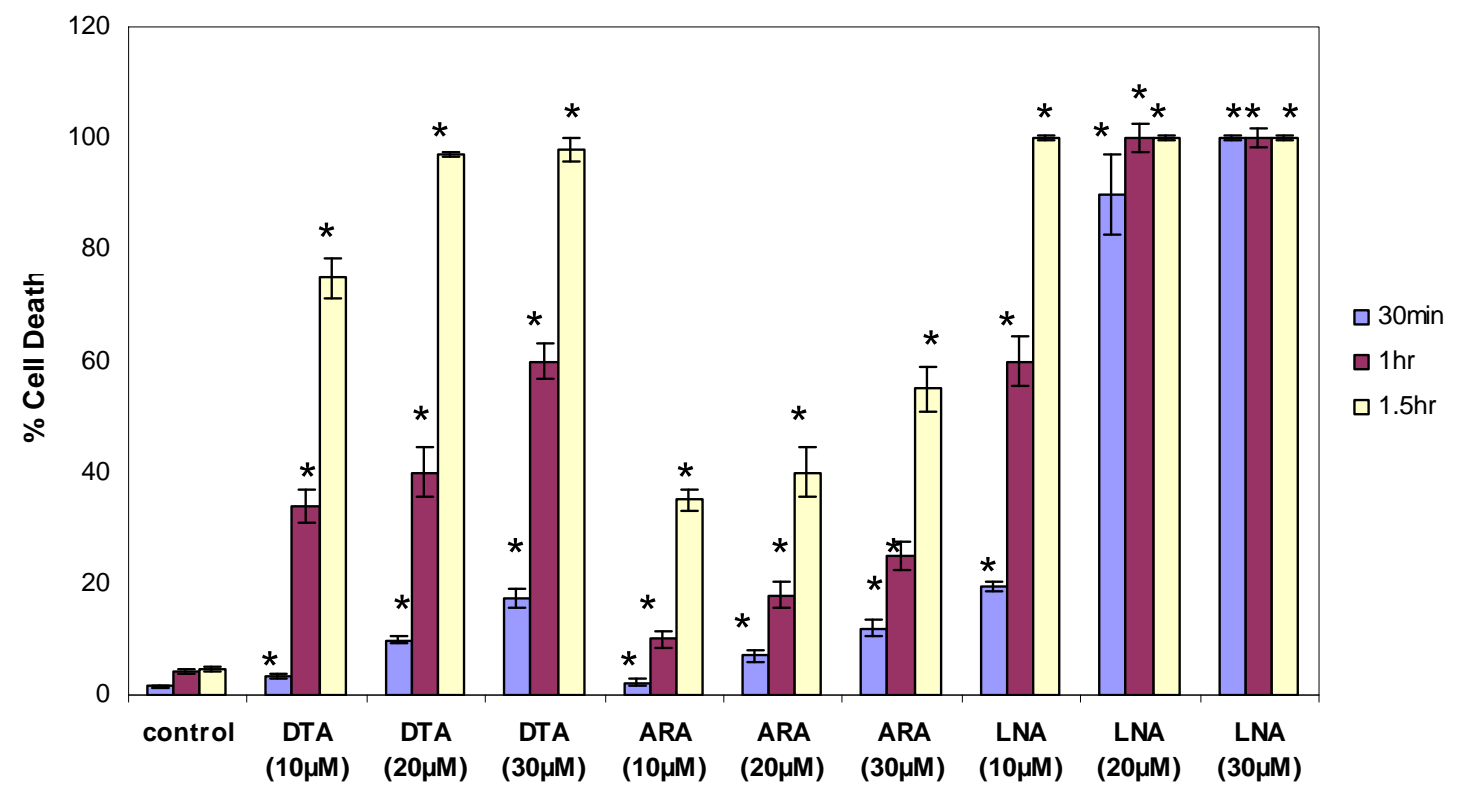

Fig. (6). Cell death resulting from incubation with omega-6 fatty acids measured as PI florescence. Thymocytes were incubated with DTA, ARA or LNA for 30min, 1 and 1.5 hrs with various concentrations. Note that the scale for cell death is different from that in Figs. (4, 5). * Significantly different from control $(\mathrm{p}<0.05)$.

heart attack [20-22]. DHA and EPA also increase membrane fluidity in aortic endothelial cells [7]. While Leaf and colleagues did not test for fluidity changes by omega- 6 fatty acids, their studies on cardiac arrhythmias [23, 24] and suppression of voltage-gated calcium [25] and sodium [26] channel currents all show that the omega- 6 fatty acids have similar actions to the omega-3s. Their studies on ionic currents as well as our current studies on fluidity show similar effects of omega- 3 and omega- 6 fatty acids.

There must be a different mechanistic explanation for the beneficial effects of fish consumption, perhaps related to there being relatively low concentrations of saturated fats in fish or effects of omega-3 fatty acids on immune system function. Omega-3 PUFAs such as DHA give rise to docosatrienes, such as neuroprotectin D1 [27, 28] and resolvin E1 [29] that are anti-inflammatory. Some of the anti-inflammatory [30] and immunosuppressive [31] actions of DHA and EPA are mediated by altering lipid raft formation [32]. Humans fed DHA show lowered levels of prostaglandin E2 and leukotriene B4 in response to administered lipopolysaccharide, reduced natural killer cell activity and reduced secretion of interleukin $1 \beta$ and TNF $\alpha$ [33]. Dietary fish oil given to mice inhibited both Th1 and Th2 responses, increased production of immunomodulatory cytokines such as IL-10 and reduced delayed-type hypersensitivity [34]. These effects on immune system function may be the more important factors underlying the benefits of fish consumption. Other possible mechanisms include induction of various kinases [35] and altered gene expression [36].

Our previous studies of membrane fluidity focused on actions of environmental pollutants. We found that orthosubstituted but not coplanar PCB congeners caused an increase in membrane fluidity than was correlated in time and concentration with rapid cell death of both neurons [37] and thymocytes [38]. We also found that low concentrations of toxaphene (10-20 ppm) caused a decrease in membrane fluidity, but still triggered significant cell death [13]. These observations indicate that membrane fluidity is very important to cell integrity, and that any acute perturbation, whether it be an increase or a decrease, can cause acute cell death. Stearic acid, a saturated C-18 fatty acid, did not exactly follow this pattern, however. Stearic acid significantly decreased membrane fluidity, consistent with previous findings [39], but did not cause marked cell death. Further study of the relative changes induced by saturated fatty acids on both fluidity and cytotoxicity is needed to understand this observation.

Our results call into question the hypothesis that the relative beneficial effects of consumption of omega- 3 as compared to omega- 6 fatty acids on cardiovascular function is due to the ability of omega-3 fatty acids to increase membrane fluidity. At least in murine thymocytes, omega- 6 fatty acids are more potent at this than omega- 3 fatty acids. Our results could reflect our use of a different cell type, but may also reflect a general rule that omega- 6 fatty acids also increase membrane fluidity. If indeed fish consumption and omega-3 fatty acids have benefits to health, there must be other mechanisms responsible.
ABBREVIATIONS
DHA $=$ Docosahexaenoic acid
$\mathrm{EPA}=$ Eicosapentaenoic
ALA $=\alpha$-linolenic acid
DTA $=$ Docosatetraenoic acid
$\mathrm{ARA}=$ Arachidonic acid
LNA $=$ Linoleic acid
$\mathrm{DPH}=1,6$-diphenyl-1,3,5-hexatriene
PUFA $=$ Polyunsaturated acid 
PI $=$ Propidium iodide

$\mathrm{P} \quad=$ Polarization

FA $=$ Fatty acid

\section{REFERENCES}

[1] Stephenson CB. Fish oil and inflammatory disease: Is asthma the next target for n-3 fatty acid supplements? Nutr Rev 2004; 62: 486-9.

[2] Harper CR, Jacobson TA. The fats of life. The role of omega-3 fatty acids in the prevention of coronary heart disease. Arch Intern Med 2001; 161:2185-92.

[3] Siddiqui RA, Shaikh SR, Sech LA, Yount HR, Stillwell W, Zaloga GP. Omega 3-fatty acids: health benefits and cellular mechanisms of action. Mini Rev Med Chem 2004; 4: 859-71.

[4] Calder PC, Yaqoob P, Thies F, Wallace FA, Miles EA. Fatty acids and lymphocyte functions. Br J Nutr 2002; 87 Suppl 1: S31-48.

[5] McNamara RK, Jandacek R, Rider T, et al. Abnormalities in the fatty acid composition of the postmortem orbitofrontal cortex of schizophrenic patients: gender differences and partial normalization with antipsychotic medications. Schizophr Res 2007; 91: 37-50.

[6] Leaf A, Xiao YF. The modulation of ionic currents in excitable tissues by n-3 polyunsaturated fatty acids. J Memb Biol 2001; 184: 263-71.

[7] Hashimoto M, Hossain S, Yamasaki H, Yazawa K, Masumura S. Effects of eicosapentaenoic acid and docosahexaenoic acid on plasma membrane fluidity of aortic endothelial cells. Lipids 1999; 34: 1297304.

[8] GISSI- Preventative Investigators. Dietary supplementation with n-3 polyunsaturated fatty acids and vitamin $\mathrm{E}$ after myocardial infarction: results of the GISSI-Prevenzione trial. Lancet 1999; 354: 447-55.

[9] $\mathrm{Hu}, \mathrm{FB}$. The role of $\mathrm{n}-3$ polyunsaturated fatty acids in the prevention and treatment of cardiovascular disease. Drugs Today 2001; 37: 49-56.

[10] Albert CM, Campos H, Stampfer MJ, et al. Blood levels of long chain $\mathrm{n}-3$ fatty acids and the risk of sudden death. N Engl J Med 2002; 346: 1113-8.

[11] Leaf A, Kang JX, Xiao YF. Fish oil fatty acids as cardiovascular drugs. Curr Vasc Pharmacol 2008; 6: 1-12.

[12] Tan Y, Chen CH, Lawrence D, Carpenter DO. Ortho-substituted PCBs kill cells by altering membrane structure. Toxicol Sci 2004; 80: 54-9.

[13] Sandal S, Yilmaz B, Chen CH, Carpenter DO. Comparative effects of technical toxaphene, 2,5-dichloro-3-biphenylol and octabromodiphenylether on cell viability, $\left[\mathrm{Ca}^{2+}\right]_{\mathrm{i}}$ levels and membrane fluidity in mouse thymocytes. Toxicol Letts 2004; 151: 417-28.

[14] Oyama Y, Carpenter DO, Ueno S, Hayashi H, Tomiyoshi F. Methylmercury induces $\mathrm{Ca}^{2+}$-dependent hyperpolarization of mouse thymocytes: a flow cytometric study using fluorescent dyes. Eur J Pharmacol 1995; 293: 101-7.

[15] Chen CH, Zuklie BM, Roth LG. Elucidation of biphasic alterations on acetylcholinesterase (AChE) activity and membrane fluidity in the structure-functional effects of tetracaine on AChE-associated membrane vesicles. Arch Biochem Biophys 1998; 351: 135-40.

[16] Lasner M, Roth LG, Chen CH. Structure-functional effects of a series of alcohols on acetylcholinesterase-associated membrane vesicles: elucidation of factors contributing to the alcohol action. Arch Biochem Biophys 1995; 317: 391-6.

[17] Calder PC, Yaqoob P, Harvey DJ, Watts A, Newsholme EA. Incorporation of fatty acids by concanavalin A-stimulated lymphocytes and the effect on fatty acid composition and membrane fluidity. Biochem J 1994; 300: 509-18.

[18] Sturlan S, Baumgartner M, Roth E, Bachleitner-Hofmann T. Docosahexaenoic acid enhances arsenic trioxide-mediated apoptosis in arsenic trioxide-resistant HL-60 cells. Blood 2003; 101: 4990-7.

[19] Siddiqui RA, Jenski LJ, Neff K, Harvey K, Kovacs RJ, Stillwell W. Docosahexaenoic acid induces apoptosis in jurkat cells by a protein phophatase-mediated process. Biochim Biophy Acta 2001; 1499: 26575 .
Leaf A, Kang JX, Xiao YF, Billman GE. Clinical prevention of sudden cardiac death by n-3 polyunsaturated fatty acids and mechanism of prevention of arrhythmias by n-3 fish oils. Circulation 2003; 107: 26324.

[21] Xiao YF, Kang JX, Morgan JP, Leaf A. Blocking effects of polyunsaturated fatty acids on $\mathrm{Na}+$ channels of neonatal rat ventricular myocytes. Proc Natl Acad Sci USA 1995; 92: 11000-4.

[22] Kang JX, Li Y, Leaf A. Regulation of sodium channel gene expression by class I antiarrhythmic drugs and $\mathrm{n}-3$ polyunsaturated fatty acids in cultured neonatal rat cardiac myocytes. Proc Natl Acad Sci USA 1997; 94: 2724-8.

[23] Kang JX, Leaf A. Protective effects of free polyunsaturated fatty acids on arrhythmias induced by lysophosphatidylcholine or palmitoylcarnitine in neonatal rat cardiac myocytes. Eur J Pharmacol 1996; 297: 97-106.

[24] Kang JX, Leaf A. Prevention of fatal cardiac arrhythmias by polyunsaturated fatty acids. Am J Clin Nutr 2000; 71: 202S-7S

[25] Xiao YF, Gomez AM, Morgan JP, Lederer WJ, Leaf A. Suppression of voltage-gated L-type $\mathrm{Ca}^{2+}$ currents by polyunsaturated fatty acids in adult and neonatal rat ventricular myocytes. Proc Natl Acad Sci USA 1997; 94: 4182-7.

[26] Xiao YF, Wright SN, Wang GK, Morgan JP, Leaf A. Fatty acids suppress voltage-gated $\mathrm{Na}+$ currents in HEK293t cells transfected with the alpha-subunit of the human cardiac $\mathrm{Na}+$ channel. Proc Natl Acad Sci USA 1998: 95: 2680-5.

[27] Bazan NG. The onset of brain injury and neurodegeneration triggers the synthesis of docosanoid neuroprotective signaling. Cell Mol Neurobiol 2006; 26: 901-13.

[28] Bazan NG. Omega-3 fatty acids, pro-inflammatory signaling and neuroprotection. Curr Opin Clin Nutr Metab Care 2007; 10: 136-41.

[29] Arita M, Yoshida M, Hong S, et al. N. Resolvin E1, an endogenous lipid mediator derived from omega-3 eicosapentaenoic acid, protects against 2,4,6-trinitrobenzene sulfonic acid-induced colitis. Proc Natl Acad Sci USA 2005; 102: 7671-6.

[30] Gil Á. Polyunsaturated fatty acids and inflammatory diseases. Biomed Pharmacother 2002; 56: 388-96.

[31] Kim W, Fan YY, Barhoumi R, Smith R, McMurray DN, Chapkin RS n-3 polyunsaturated fatty acids suppress the localization and activation of signaling proteins at the immunological synapse in murine CD4+ T cells by affecting lipid raft formation. J Immunol 2008; 181: 6236-43.

[32] Stulnig TM, Huber J, Leitinger N, et al. Polyunsaturated eicosapentaenoic acid displaces proteins from membrane rafts by altering raft lipid composition. J Biol Chem 2001; 376: 37335-40.

[33] Kelley DS, Taylor PC, Nelson GJ, et al. Docosahexaenoic acid ingestion inhibits natural killer cell activity and production of inflammatory mediators in young healthy men. Lipids 1999; 34: 31724.

[34] Sierra S, Lara-Villoslada F, Comalada M, Olivares M, Xaus J. Dietary fish oil n-3 fatty acids increase regulatory cytokine production and exert anti-inflammatory effects in two murine models of inflammation. Lipids 2006; 41: 1115-25.

[35] du Toit-Kohn JL, Louw L, Engelbrecht AM. Docosahexaenoic acid induces apoptosis in colorectal carcinoma cells by modulating the P13 kinase and p39 MAPK pathways. J Nutr Biochem 2009; 20: 106-14.

[36] Kitajka K, Sinclair AJ, Weisinger RS, et al. Effects of dietary omega-3 polyunsaturated fatty acids on brain gene expression. Proc Natl Acad Sci USA 2004; 101: 10931-6.

[37] Tan Y, Song R, Lawrence D, Carpenter DO. Ortho-substituted but not coplanar PCBs rapidly kill cerebellar granule cells. Toxicol Sci 2004; 79: 147-56.

[38] Yilmaz B, Sandal S, Chen CH, Carpenter DO. Effects of PCB 52 and PCB 77 on cell viability, $\left(\mathrm{Ca}^{2+}\right) \mathrm{i}$ levels and membrane fluidity in mouse thymocytes. Toxicology 2006;217: 184-93.

[39] Stubbs CD, Smith AD. The modification of mammalian membrane polyunsaturated fatty acid composition in relation to membrane fluidity and function. Biochim Biopys Acta 1984; 779: 89-137. 